\title{
Commentaires sur le choix des coefficients de réaction pour le calcul des écrans de soutènement
}

\section{B. SIMON}

Terrasol

Immeuble Helios

72, avenue Pasteur 93108 Montreuil Cedex
Le coefficient de réaction n'est, en aucune façon, une caractéristique intrinsèque d'un massif ou d'une couche, mais un paramètre qui tend à qualifier, de manière approchée, l'interaction entre un élément de structure et son massif de fondation pour un chargement donné. A travers le rappel de quelques-unes des recommandations traitant du choix de ce coefficient, notamment dans le cas des écrans de soutènement (Terzaghi, Ménard), l'article s'attache à dégager un certain nombre de principes essentiels pouvant aider à un choix raisonné de ce paramètre. Un ensemble de règles pratiques relatives aux ouvrages de soutènement, fondées sur la pratique acquise comme bureau d'études, est proposé en cohérence avec les formulations générales d'évaluation du tassement sous des semelles (élasticité et méthode pressiométrique) et les différents mécanismes d'interaction qui ont été identifiés.

\section{Comments relating to the choice of the coefficient of subgrade reaction for the design of earth retaining structures}

The coefficient of subgrade reaction is by no mean an intrinsic property of a soil mass or soil layer. Instead it provides a practical means for calculating, in an approximate manner and for a given loading, the interaction between a structural element and its foundation soil. This paper presents the basic principles essential in making a rational choice ot the coefficient of reaction, in reference to the recommandations pertaining to the assessment of this property, especially those for earth retaining structures (Terzaghi or Menard). A set of practical rules for earth retaining structures is provided, based on our experience gained in the design of such structures, and consistent with the general methods for estimating settlements below spread footings (elasticity or pressuremeter method) as well as the various interaction mechanisms. 


\section{Introduction}

En France, le calcul des écrans de soutènement fait largement appel à la notion de coefficient de réaction pour la vérification de l'équilibre interne de l'écran et des sollicitations créées dans les différents éléments de structure qui le composent. Cette situation tient pour une large part à la diffusion qu'ont eue durant ces vingt dernières années les programmes de poutre sur appuis élastiques incorporant des lois de réaction non linéaires pouvant caractériser les seuils de poussée et butée des différentes couches le long de l'écran. Cette situation explique que ces calculs soient souvent directement pris en charge par le bureau de structure qui, faute d'une réelle compétence géotechnique, recherchera les documents lui permettant de choisir l'ensemble des paramètres dont il a besoin, au premier rang duquel la valeur du coefficient de réaction. Celui-ci est rarement défini dans le rapport d'étude géotechnique ou dans les pièces contractuelles du marché. Le choix qui en est fait va ainsi souvent reposer sur une interprétation plus ou moins licite et plus ou moins raisonnée des quelques règles qui ont été proposées sur ce sujet (Terzaghi, Ménard et Bourdon, Chadeisson, Balay, etc.). Cet article a pour but d'éclairer sur un certain nombre de points ce concept de coefficient de réaction, afin d'identifier les principes à respecter lors du choix des valeurs utiles à ce mode de calcul. Il s'appuie sur l'expérience acquise dans la conception d'ouvrages de soutènement et celle tirée de plusieurs expertises que nous avons eues à connaitre.

\section{2}

\section{Concept de coefficient de réaction: ses limites et sa justification dans un cas particulier}

\section{1}

A travers le concept de coefficient de réaction, le projeteur recherche une équivalence entre l'interaction d'un élément de structure reposant sur un massif et celle du même élément de structure reposant sur un ensemble continu de ressorts dont la réaction $p$ (dimension d'une contrainte) est proportionnelle au déplacement y de l'interface (Fig. 1):

$$
p=k \cdot y
$$

Le rapport de proportionalité $\mathrm{k}$ (dimension $\mathrm{ML}^{-2} \mathrm{~T}^{-2}$; $\mathrm{kN} / \mathrm{m}^{3}$ en unités SI) est défini comme le coefficient de réaction de la fondation. Il est généralement supposé indépendant de la pression appliquée et de valeur constante sous la fondation. Ce modèle est dénommé modèle de Winkler.

Dans le massif, le déplacement y dépend des réactions $\mathrm{p}$ appliquées à la surface. Ce système de réactions doit être distingué du système des efforts appliqués à l'élément de fondation considéré (Fig. 2): ces deux systèmes ne sont identiques que si on suppose la fondation infiniment souple (sans rigidité propre). Dans tous les autres cas, la fondation assure une redistribution des efforts à la surface du massif. Cette redistribution résulte des conditions suivantes:
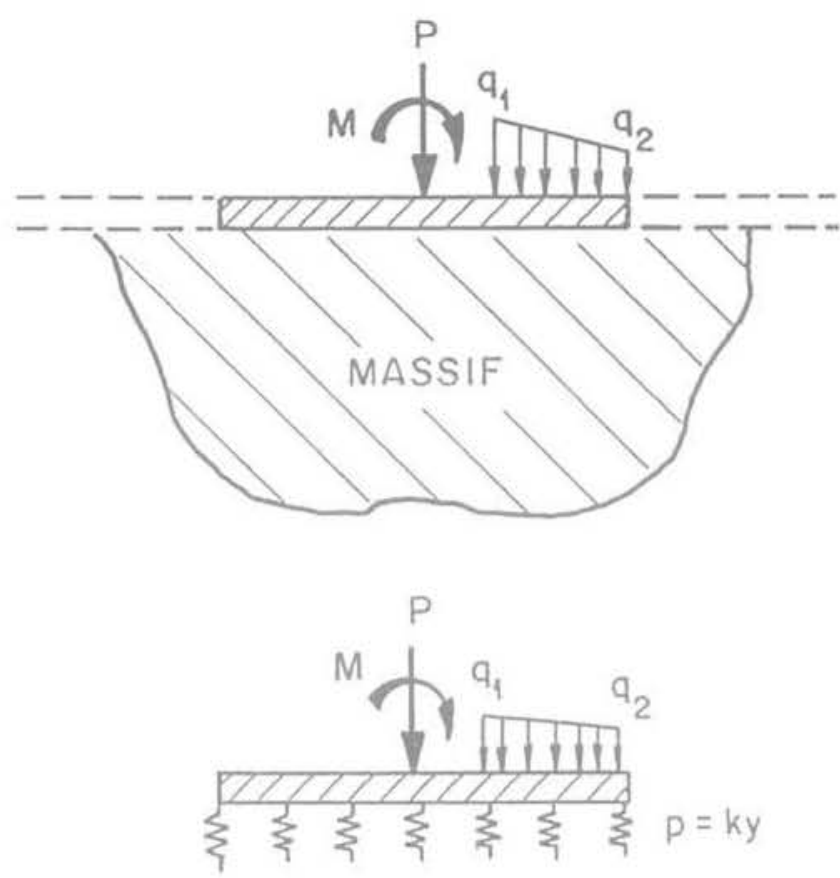

FIG.1 Principe d'équivalence fondant le concept de coefficient de réaction.

Basic concept of the coefficient of subgrade reaction.

- la structure est en équilibre sous les actions [ $\Sigma$ ] (P, M, q) et les réactions $-[\Sigma](-p)$ qui lui sont appliquées; - la déformée de la structure soumise aux efforts globaux $[\Sigma]-[\Sigma]$ est égale à celle du massif soumis aux efforts $\left[\Sigma_{i}\right]$, partout où il n'y a pas décollement.

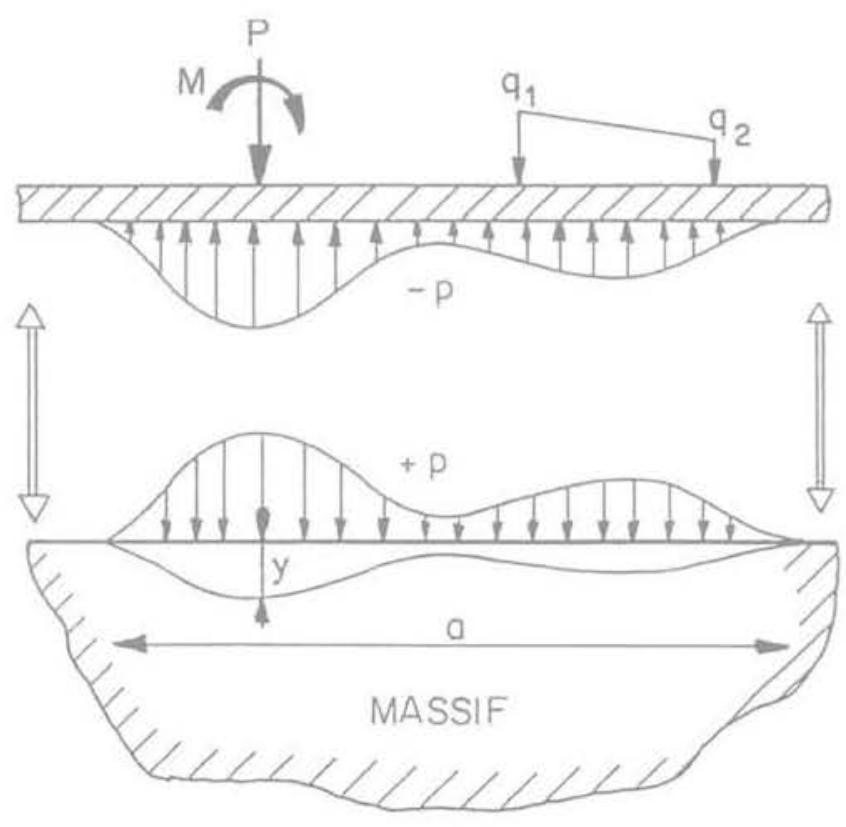

FiG. 2 Système des actions et réactions agissant sur la fondation.

System of loads and soil reactions acting on a foundation.

La déformée y du massif dépend de la largeur (a) sur laquelle sont mobilisées les réactions du massif. Cette largeur est fonction de la rigidité relative de la structure par rapport au massif qui la supporte. 
Ces deux points s'avèrent fondamentaux pour le concept de coefficient de réaction:

- ce paramètre n'est pas une propriété intrinsèque d'un massif ou d'une couche.

- c'est un paramètre d'interaction entre une structure et sa fondation;

- il n'est défini que pour un chargement donné.

Le paramètre $\mathrm{k}$ destiné à représenter de manière approchée cette interaction dépendra ainsi de la largeur (a) sur laquelle sont mobilisées les réactions du massif pour le chargement considéré et de la rigidité relative de la fondation par rapport au massif.

\section{2}

Les limitations inhérentes au concept de coefficient de réaction ont été maintes fois soulignées. Elles apparaissent clairement sur les cas limites de la figure 3.

q constant
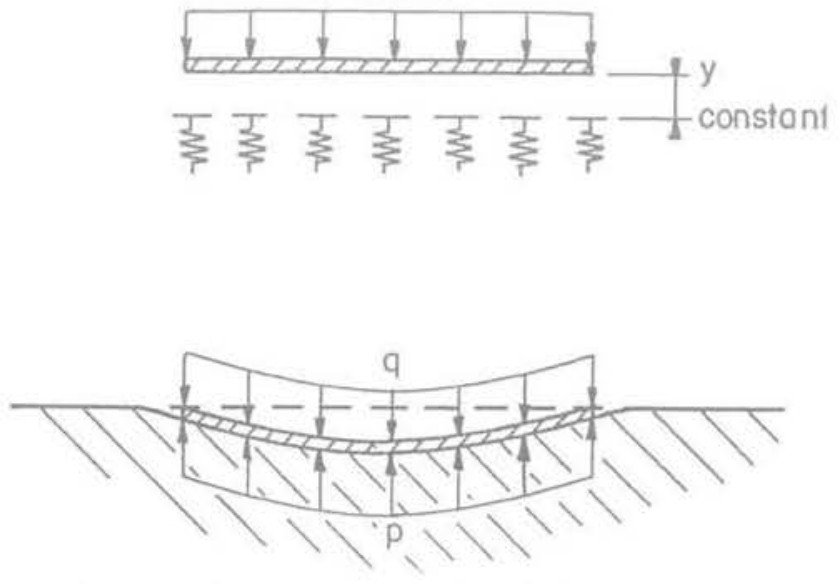

$\frac{\text { a) Cas limite 1: semelle infiniment }}{\text { souple }}$
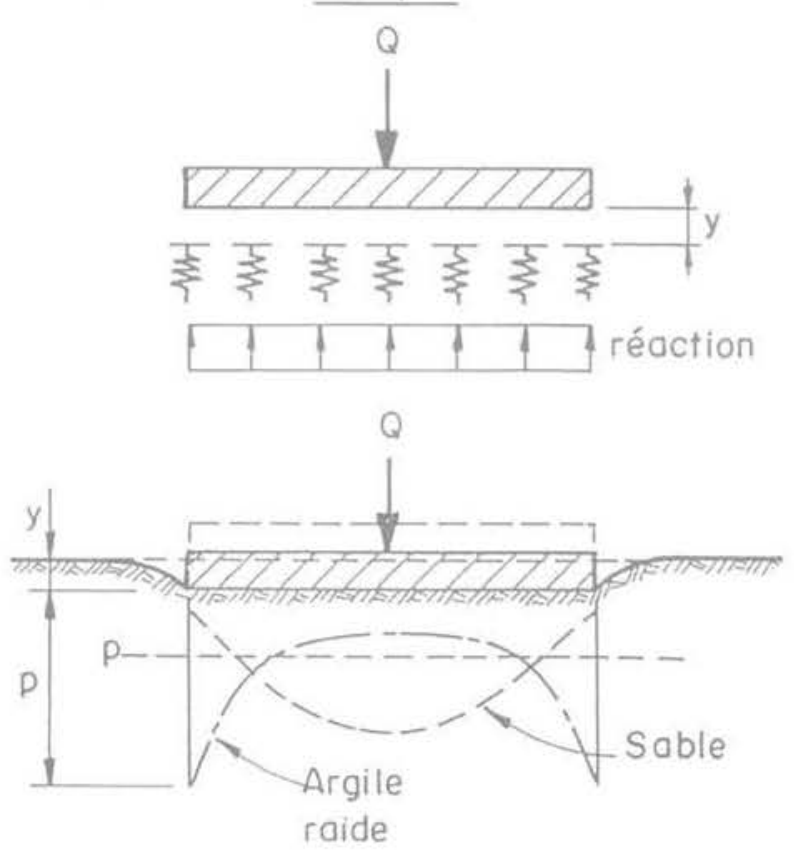

b) Cas limite 2 : semelle infiniment rigide

FiG. 3 Limitations inhérentes au concept de coefficient de réaction.

Inherent limitations in the concept of the coefficient of reaction.
Figure $3 a$-semelle infiniment souple soumise à une charge répartie uniforme: le modèle du coefficient de réaction fournit un déplacement uniforme sous la fondation alors que le modèle du massif subit un déplacement plus important au centre qu'aux bords. Ceci illustre l'incapacité du modèle à ressorts à représenter correctement l'interaction existant entre les tranches verticales du massif.

Figure $3 b$-semelle infiniment rigide soumise à une charge verticale centrée: le modèle du coefficient de réaction établit une répartition uniforme des réactions sous la semelle, alors que la distribution des réactions obtenue dans le modèle du massif ne l'est pas (réactions plus élevées à proximité des arêtes sil le massif est élastique - cas d'une argile raide - ou réactions plus faibles si le massif possède un seuil de plasticité -cas d'un massif de sable-).

D'autres limitations peuvent être rencontrées: la méthode du coefficient de réaction est inapplicable à l'estimation des sollicitations induites par des déformations résultant de facteurs autres que les charges directement appliquées à l'élément de structure (tassement dû à des charges extérieures, gonflement d'une couche sous la fondation ou relaxations dues à des variations de la nappe baignant la fondation...).

\section{3}

Il existe cependant un cas où l'équivalence entre le modèle d'un massif continu et le modèle de Winkler peut être établie de manière théorique; il s'agit du problème de la poutre de largeur b et de grande longueur, posée sur un massif élastique et soumise à une charge concentrée (Fig. 4). La solution initiale due à Biot a été développée par Vesic qui en a tiré des approximations numériques à moins de $2 \%$ du déplacement $\mathrm{y}(\mathrm{x})$, du moment fléchissant, de l'effort tranchant et de la réaction $\mathrm{P}(\mathrm{x})$ sous la poutre. Il est remarquable que le rapport entre les expressions de $\mathrm{P}(\mathrm{x})$ et $\mathrm{y}(\mathrm{x})$ ainsi établies est trouvé indépendant de $\mathrm{x}$; il ne dépend que des seuls paramètres dimensionnels définissant la section de poutre. L'hypothèse de Winkler se trouve ainsi pratiquement satisfaite dans ce cas particulier.

On notera en outre que sur la base de l'équivalence relevée entre les solutions obtenues par les deux modèles, Vesic a proposé l'expression suivante du coefficient de réaction conduisant à des résultats proches de ceux du massif élastique:

$$
k=\frac{0.65}{b} \cdot 12 \sqrt{\frac{E_{s} b^{4}}{E_{b} I}} \cdot \frac{E_{s}}{1-v_{s}^{2}}
$$

avec: $E_{5}, v_{5}$ module d'Young et coefficient de Poisson du massif;

$\mathrm{E}_{b} \mathrm{I}$ produit d'inertie de la poutre;

b largeur de la poutre.

L'approximation de la solution obtenue pour la poutre infinie est encore bonne pour des longueurs réelles de poutre $L$ supérieures ou égales à 2,25 $\mathrm{I}_{0}$ avec:

$$
1_{0}=\cdot \sqrt[4]{\frac{4 E_{\mathrm{b}} I}{\mathrm{~kb}}}
$$

Vesic montre également que les solutions obtenues par la méthode du coefficient de réaction surestiment les moments fléchissants dans la poutre (de l'ordire de $6 \%$ en moyenne) mais sous-estiment les déplacements 


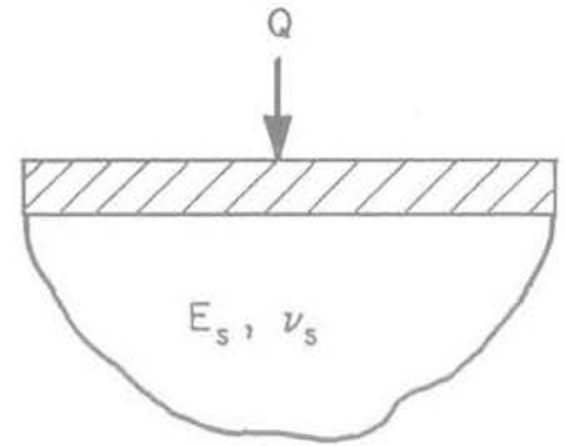

Intégration numérique

$y, y^{\prime}, M, \uparrow$

(Vesic, 1961 )
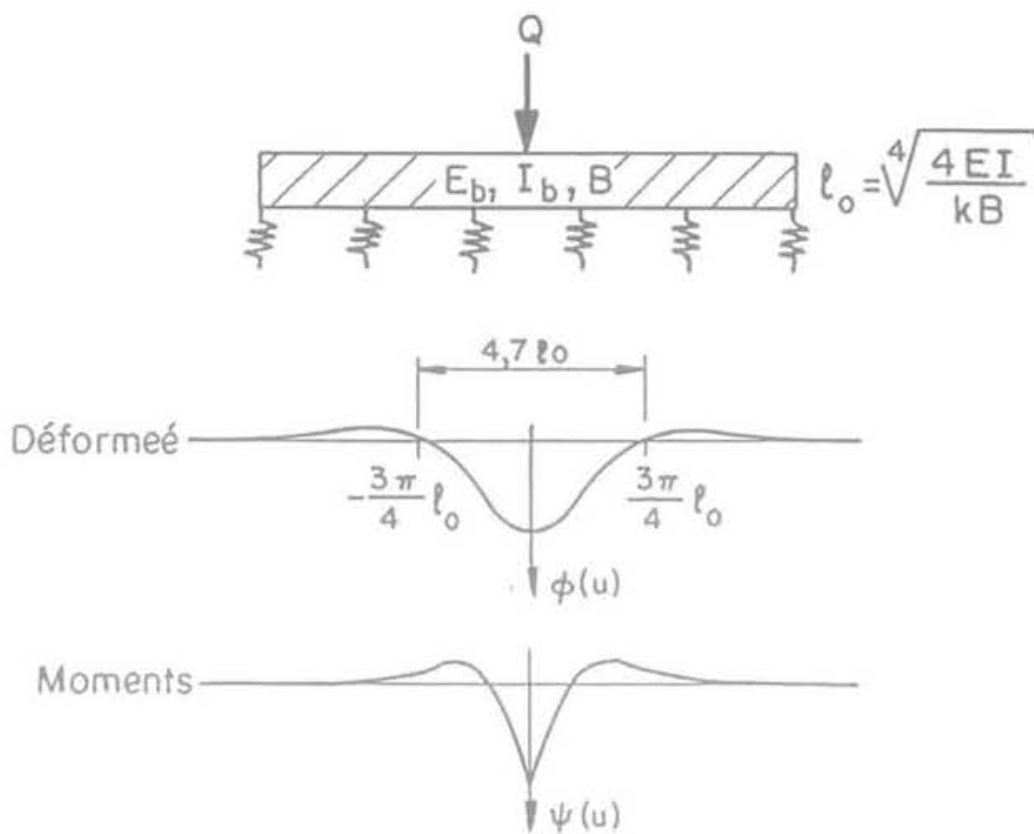

Bonne concordance entre les deux solutions:

$$
k=\frac{0,65}{B} \sqrt[12]{\frac{E_{s} B^{4}}{E_{b} I_{b}}} \cdot \frac{E_{s}}{\left(1-\nu_{s}^{2}\right)}
$$

FG 4 Analogie des solutions obtenues pour une poutre de grande longueur soumise à une charge concentrée. Similarity between the solutions for a long beam subjected to a concentrated loading.

et les réactions (environ-10\%). La méthode du coefficient de réaction est donc valable au plan pratique pour ces différents cas de chargement.

\section{Remarque:}

On note la proportionalité de $\mathrm{k}$ avec les facteurs $E_{\mathrm{s}}{ }_{\mathrm{s}}^{1,023}$ et $\mathrm{E}_{\mathrm{b}} \mathrm{I}^{-0,083}$ dans la formule (2). Biot dans sa publication initiale avait cherché une équivalence entre les deux modèles sur la seule base du moment maximal obtenu dans la poutre. Cela le conduisait à une expression où $k$ variait avec les facteurs $E_{s}^{i, 11}$ et $E_{b} I^{-0,11}$. Ceci permet de souligner, puisque la notion de coefficient de réaction est basée sur la recherche d'une équivalence entre deux modèles que, cette équivalence peut être cherchée par ajustement soit de la déformée soit de la valeur maximale du moment fléchissant; les deux ajustements peuvent conduire à des valeurs $\mathrm{k}$ sensiblement différentes. Il appartient donc au projeteur de définir la finalité de son calcul pour orienter le choix du coefficient de réaction.

\section{3}

\section{Quelques constatations permettant de bâtir un cadre de référence}

\section{1}

Dans son article de référence de 1955, Terzaghi a proposé, sur la base des valeurs expérimentales qu'il avait recueillies, des relations empiriques caractérisant le coefficient de réaction contre différents types d'ouvrages. La comparaison de ces relations d'ordre semi-expérimental aux solutions déduites de la théorie de l'élasticité permet d'éclairer un certain nombre de points.

La théorie de l'élasticité isotrope établit le tassement $y$ d'une plaque rectangulaire à la surface d'un massif élastique semi-infini $\mathrm{E}_{s}, v_{\mathrm{s}}$ :

$$
y=c_{p} p R \frac{\left(1-v_{s}^{2}\right)}{E_{s}}
$$

p contrainte moyenne répartie;

$\mathrm{R}$ demi petit côté du rectangle;

$\mathrm{C}_{\text {r }}$ coefficient de forme fonction de la position du point et de l'élancement du rectangle (Caquot et Kérisel, 1956). Pour une plaque rigide $B \times B: C_{r}=$ 1,76 , pour un rectangle rigide $\mathrm{B} \times 10 \mathrm{~B}: \mathrm{C}_{7}=4,17$.

Appliqué au cas d'une argile surconsolidée pour laquelle on peut retenir une valeur du module d'Young drainé $\mathrm{E}_{\mathrm{s}}=100 \mathrm{c}_{\mathrm{u}}$ ( $\mathrm{c}_{\mathrm{v}}$ : cohésion non drainée) et un coefficient de Poisson $v_{\mathrm{s}}=0,2$, ces relations conduisent aux expressions suivantes du coefficient de réaction:

$$
k_{B, B}=120 \frac{C_{u}}{B} \text { et } k_{B, 10 B}=50 \frac{C_{u}}{B}
$$

Ces expressions sont proches des relations empiriques proposées par Terzaghi pour les mêmes configurations:

$$
k_{B, B}=100 \frac{c_{u}}{B} \text { et } k_{B, 10 B}=\frac{200}{3} \frac{c_{u}}{B}
$$


Cela démontre l'intérêt de la théorie de l'élasticité pour rendre compte du sens des variations du coefficient de réaction avec les dimensions de la plaque considérée. Il est remarquable également que les résultats auxquels celle-ci conduit confirment, dans le cas particulier considéré, les ordres de grandeur expérimentaux rapportés par Terzaghi.

Terzaghi a proposé de rapprocher le cas de l'écran vertical souple de celui constitué par l'écran réel et son symétrique (Fig. 5). En supposant le massif non pesant, le problème s'identifierait au choix du coefficient de réaction pour un écran de largeur $\mathrm{B}=2 \mathrm{a}$. Terzaghi a fait cependant observer que le refoulement (déplacement vertical ascendant) à la surface du massif réel conduisait à des déformations de l'écran supérieures à celle du problème équivalent considéré (où le déplacement vertical de la limite Oc est nul). Le coefficient de réaction est ainsi plus faible que celui qui serait obtenu en négligeant cet effet de surface: Terzaghi propose de retenir la formulation de l'équation (6) avec $\mathrm{B}=3$ a au lieu de $\mathrm{B}=2 \mathrm{a}$.

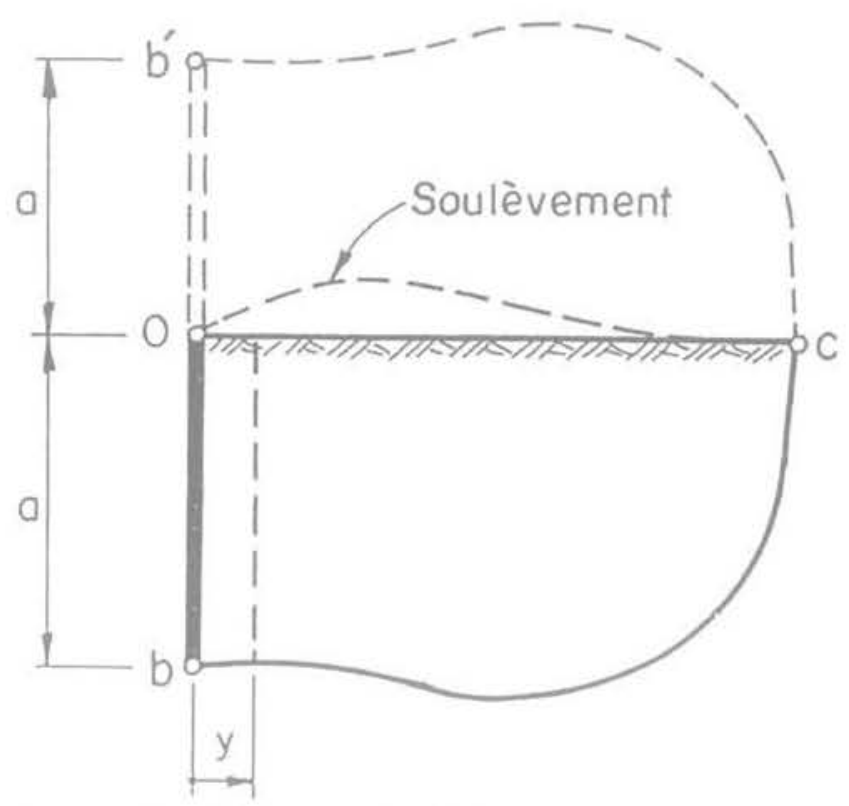

FG.5 Écran vertical, influence du soulèvement de la surface libre.

Vertical wall, influence of the heave at the unconfined soil surface.

Ceci le conduit aux recommandations de la figure 6 pour le coefficient de réaction contre un écran vertical souple placé contre une argile raide. On observera la distinction faite pour la partie inférieure en contrebutée qui fait assimiler le coefficient de réaction à celui d'une bande de largeur égale à la hauteur de contrebutée $\left(B=D^{\prime \prime}\right)$. Aucune influence de la surface libre n'est ainsi prise en compte dans cette zone où les déformations du massif demeurent parfaitement confinées.

\section{2}

La méthode pressiométrique établit également le tassement sous une semelle rigide (Ménard et Rousseau, 1962):

$$
y=p \cdot\left(\frac{2}{9 E_{m}} B_{0}\left(\lambda_{d} \frac{B}{B_{0}}\right)^{\alpha}+\frac{\alpha}{9 E_{m}} \lambda_{c} B\right)
$$

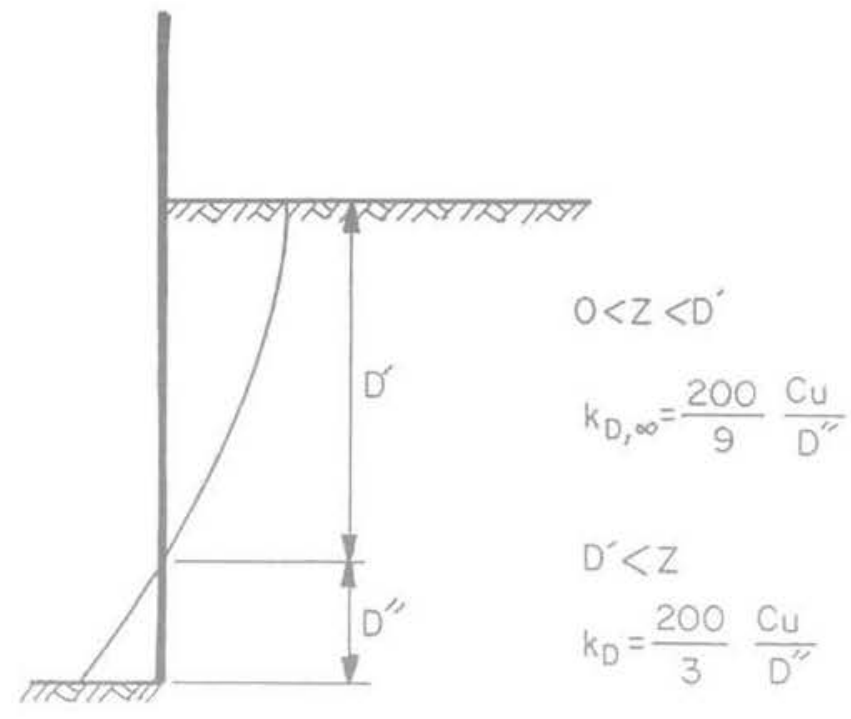

Fig. 6 Règles relatives aux écrans souples de soutènement (Terzaghi, 1955).

Rules pertaining to flexible retaining structures (Terzaghi, 1955).

$\mathrm{E}_{\mathrm{m}}$ module pressiométrique du massif homogène équivalent:

$\alpha \quad$ coefficient rhéologique;

$\mathrm{B}_{0}$ dimension de référence $\left(\mathrm{B}_{0}=0,6 \mathrm{~m}\right)$;

$\lambda, \lambda_{\mathrm{d}}$ coefficients de forme fonction de l'élancement de la fondation (pour un rectangle $\mathrm{B} \times 20 \mathrm{~B}: \lambda_{\mathrm{c}}: 2,65$ et $\lambda_{\mathrm{d}}=1,5$ ).

L'application de cette relation fondamentale au cas de l'écran fictif de la figure 5 (assimilé à un rectangle de grand élancement horizontal et de largeur $\mathrm{B}=2$ a) établirait l'expression suivante du coefficient de réaction $\mathrm{k}=\mathrm{p} / \mathrm{y}$ :

$$
k=\frac{E_{m}}{0,13(8,8 a)^{\alpha}+\alpha \frac{a}{3}}
$$

Cette expression, il faut le noter, est formellement identique à celle proposée par Ménard pour estimer le coefficient de réaction horizontale devant un pieu de rayon a. Elle ne s'identifie cependant pas à celle spécifique proposée pour les écrans de soutènement (Ménard et Bourdon, 1964), le long de la partie en fiche:

$$
k=\frac{E_{m}}{0,13(9 a)^{\alpha}+\alpha \frac{a}{2}}
$$

Ménard et Bourdon invoquent en effet l'effet de compression simple qui se manifeste devant l'écran par le refoulement de la surface du sol. Selon eux, cet effet est représenté par le coefficient $1 / 2$ appliqué à la contribution sphérique du tassement (au lieu du coefficient $1 / 3$ applicable au cas général). Ceci conduit ainsi à une valeur du coefficient de réaction plus faible que celle obtenue par la formule générale applicable à des situations de chargement avec confinement latéral. Cette correction doit être rapprochée de celle faite par Terzaghi pour les écrans. L'une et l'autre montrent donc l'influence des conditions aux limites (ici position de la surface libre du massif) sur la détermination du coefficient de réaction.

En complément il faut relever que Ménard et Bourdon identifient le paramètre géométrique (a) à la dis- 
tance entre le fond de fouille et le centre de rotation du pied du rideau. Ils précisent que dans le cas général cette hauteur est de l'ordre des $2 / 3$ de la section enterrée h du rideau; ce n'est que dans le cas d'un écran très rigide ne développant aucun effort de contre-butée que cette grandeur s'identifie à la hauteur totale $(\mathrm{a}-\mathrm{h})$.

\section{Remarque :}

Il serait possible de discuter l'assimilation directe de (a) avec la distance séparant le fond de fouille du centre de rotation. En effet ceci revient à poser l'équivalence stricte entre la déformée du rideau sur cette hauteur avec le tassement sous une fondation de même demilargeur. Ceci néglige l'effet de cuvette provoqué par une fondation: le tassement ne s'annule pas au bord de la fondation mais à un point situé plus à l'extérieur. La déformée du rideau s'annulant ainsi à une distance (a) de l'axe de symétrie considéré, cette déformée ne peut être attribuée qu'à une fondation de demi-largeur inférieure à (a) Cette remarque est encore plus forte si on observe que la formule de tassement prise comme référence s'applique à des fondations rigides sous lesquelles le tassement est a priori constant. Une meilleure approche pourrait donc être celle attribuant la déformée observée sur une largeur totale L à une semelle équivalente de largeur $B$ inférieure à $L$. Les résultats de l'élasticité permettraient de retenir une relation telle que :

$$
B=\frac{L}{1,5}
$$

Ceci conduirait à augmenter sensiblement les valeurs $k$ obtenues dans la zone en fiche (conduisant dans le cas général à $\mathrm{a}=4 / 9 \mathrm{~h}$ au lieu de $\mathrm{a}=2 / 3 \mathrm{~h}$ ). Cet amendement serait également conforme au sens des écarts relevés entre les observations expérimentales et les estimations de déformées déduites des règles actuelles (Schmitt, 1984).

\section{4}

\section{Règles pratiques pour le choix du coefficient de réaction le long d'un soutènement}

\section{1}

Les différentes observations rappelées ci-dessus et les quelques réflexions qui ont été présentées justifient le cadre général suivant que nous adoptons pour le choix des coefficients de réaction le long des écrans de soutènement. Celui-ci reprend en partie les recommandations faites par le LCPC (Balay, 1984) mais les amendes sur plusieurs points.

(Fig. 7):

Différentes zones sont tout d'abord distinguées - celles où la surface libre est intéressée: 1-zone en poussée ou 2-zone en butée;

- celles où la surface libre n'est pas intéressée (déformations entièrement confinées) : 3-partie à l'amont du soutènement placée entre un niveau d'ancrage et un encastrement en pied, ou entre deux niveaux d'ancrage, 4-partie placée au droit d'un appui (tirant précontraint ou buton).

\section{Surface libre intéressée}

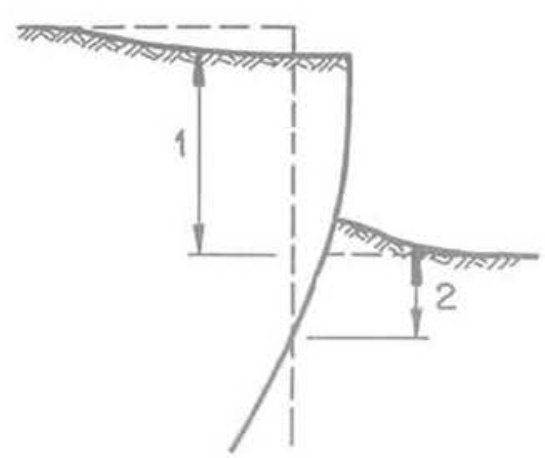

Surface libre non intéressée

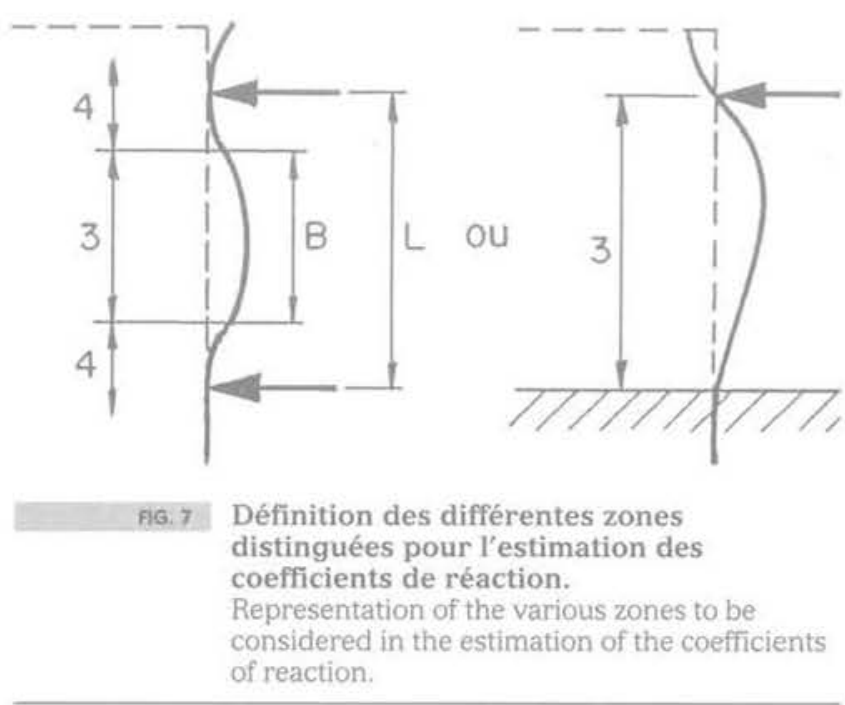

\section{La surface libre est intéressée}

Zone 2 (en butée) : La formulation de Ménard et Bourdon (1964) est directement applicable (équation 9). Le paramètre (a) est choisi sur la base des propositions de Balay (1984) (Fig. 8). Il peut être souhaitable de vérifier à l'issue du calcul au coefficient de réaction que le mode de déformation de la paroi et la position du centre de rotation de la partie en fiche s'accordent bien avec la position que représentent implicitement les règles définissant (a). Dans certains cas il pourra être tenu compte de la remarque faite concernant l'assimilation de (a) aux 2/3 de la distance (d) entre le fond de fouille et le point de déplacement nul s'il existe : $(\mathrm{a}=$ $4 / 9$ d).

Zone 1 (en poussée) : Les recommandations de Balay font extrapoler à cette partie la formulation donnée initialement par Ménard pour la seule partie en fiche et proposent une règle pour le choix du paramètre (a). La pertinence de ce choix peut également être vérifiée notamment à l'issue de la première phase de calcul où le soutènement travaille souvent en console. La remarque faite concernant la relation entre (a) et les $2 / 3$ de la longueur d'écran dont la déformée est notable peut également trouver une application. La figure 9 fournit un exemple des variations du coefficient $k$ avec la hauteur des passes terrassées dans une argile de consistance moyenne: la valeur k décroît rapidement 


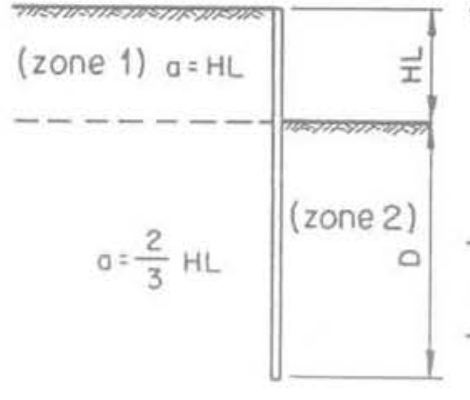

$H L<D$

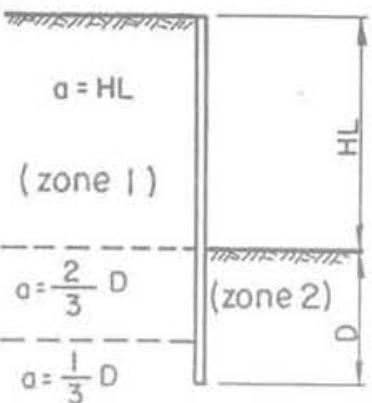

(2) $H L>D$

Choix du paramètre géométrique «a » (Balay, 1984).

Choice of the geometrical parameter \& a (Balay, 1984).

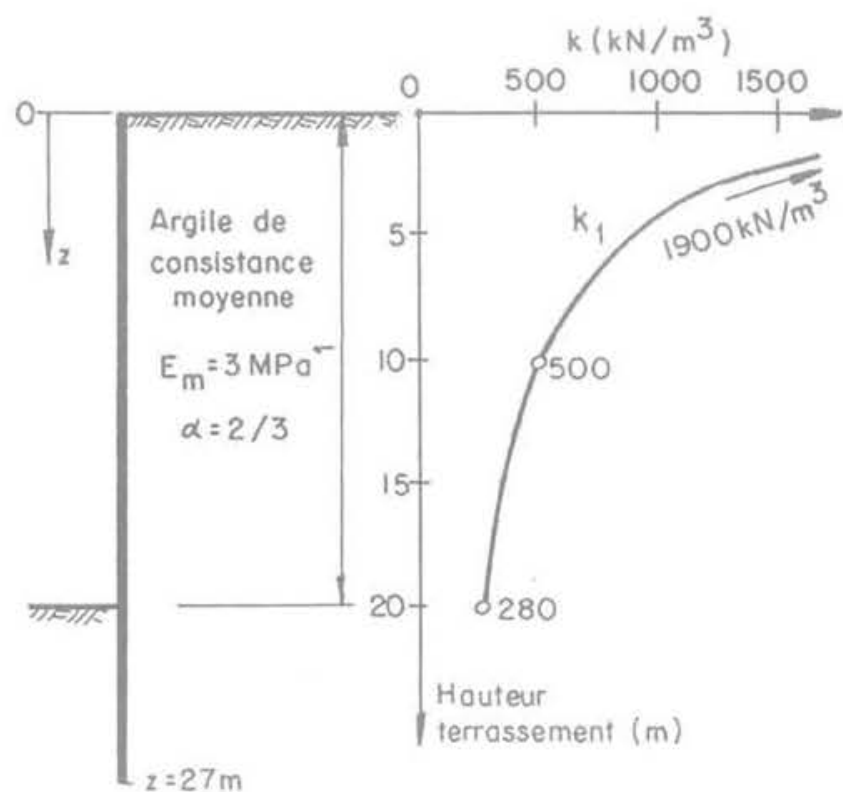

FIG.9 Exemple des variations du coefficient de réaction obtenues dans la zone [1].

Variations in the coefficient of reaction within zone [1].

avec la hauteur terrassée; pour une première passe de terrassement de $2 \mathrm{~m}$ la valeur est plus de 6 fois supérieure à celle applicable lorsque $20 \mathrm{~m}$ se trouvent terrassés (et qu'aucun butonnage n'est mis en place).

\section{3}

\section{La surface libre n'est pas intéressée}

Zone 3: Aucune règle n'est donnée par Balay (1984). En observant que les déformations demeurent confinées, il est justifié de faire référence à l'expression générale du tassement sous la semelle de largeur B (obtenue par rotation de la paroi de $90^{\circ}$ ) et à la constatation qu'une fondation de largeur B provoque une cuvette de déformation sur une largeur L voisine de 1,5B (Fig. 10). Cette largeur peut être identifiée à la distance entre les points d'appuis (ou le point d'appui supérieur et le niveau d'encastrement inférieur). Cette assimilation entre la bande d'écran de largeur $B=L / 1,5$ et une semelle équivalente conduit à une expression du

coefficient de réaction équivalente à la relation (8) puisque $B=2 a$ :

$$
k=\frac{E_{m}}{0,13(4,4 B)+\frac{\alpha B}{6}}
$$
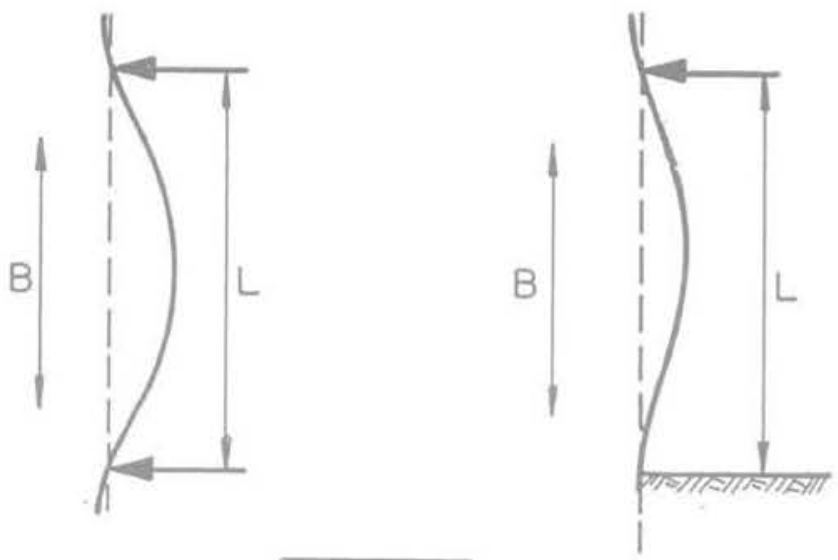

$$
B \simeq \frac{L}{1,5}
$$

FG,10 Largeur B d'une semelle donnant une déformée semblable à celle obtenue sur la surface de longueur $\mathrm{L}$.

Width $\mathrm{B}$ of a footing giving similar

displacements to those obtained on a surface of length $\mathrm{L}$.

Zone 4 : Le coefficient de réaction applicable au droit des appuis doit être choisi en référence à la solution établie par le modèle de Winkler pour la poutre de longueur infinie et de largeur b soumise à une charge concentrée (Fig. 11). Cette solution fait référence à la longueur de référence $l_{0}$ fonction de la raideur $k$ des appuis (relation 3).

Les déplacements de la poutre sont localisés sur l'intervalle centré sur le point d'application de la charge et de longueur $(3 \pi / 2) 1_{0}$, soit une largeur sollicitée proche de $\mathrm{L}=4,51_{0}$.

Dans le modèle du massif, cette déformée peut être assimilée à celle provoquée par une semelle de largeur $\mathrm{B}=\mathrm{L} / 1,5$ soit $\mathrm{B}=3 \mathrm{I}$, pour laquelle la relation (10) fournit une estimation du coefficient de réaction sous la semelle.

Ceci fait rechercher la compatibilité entre une valeur de $k$ choisie a priori pour estimer $l_{0}$ et celle fournie par la relation (10) appliquée à une semelle de largeur $\mathrm{B}=3 \mathrm{l}_{\circ}$. Cette résolution est itérative selon le processus décrit sur la figure 11. Dans la pratique, la convergence est rapide (deux ou trois calculs suffisent). La méthode proposée fournit ainsi la valeur du module de réaction applicable de part et d'autre des appuis (butons ou tirants) compatible avec la rigidité propre de la paroi. Cette valeur est applicable à la zone $[-1,5]_{\text {, }}$ $\left.+1,51_{0}\right]$ de l'écran centrée sur l'appui considéré.

Cette méthode n'est plus applicable quand l'intervalle ci-dessus inclut la surface supérieure du massif. Une adaptation peut alors être recherchée en privilégiant alors la relation (9).

Dans l'exemple de la figure 9 , le coefficient de réaction pour un terrassement de $20 \mathrm{~m}$ est voisin de $300 \mathrm{kN} / \mathrm{m}^{3}$ en négligeant les appuis. En partant de cette 

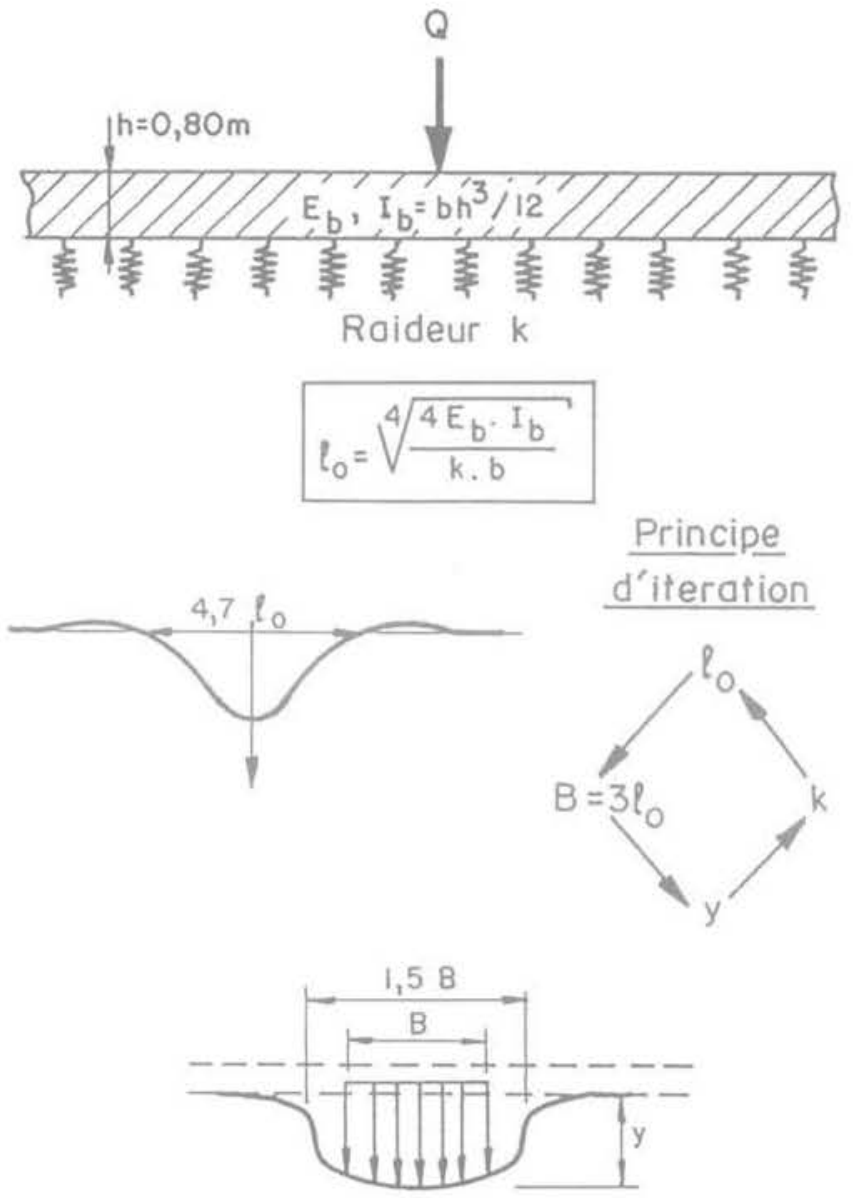

$B \simeq 3 ?_{0}$

FIG.11 Principe du mode itératif de détermination du coefficient de réaction applicable au droit d'une charge concentrée (zone 4).

Principle for the iterative method of determining the coefficient of reaction under a concentrated loading (zone 4).

valeur le processus itératif décrit établit successivement (paroi : épaisseur $1 \mathrm{~m}$, module $\mathrm{E}_{\mathrm{h}}=22000 \mathrm{MPa}$ ).

\begin{tabular}{c|c|c|c}
\hline Itération & $k_{0}\left(\mathrm{KN} / \mathrm{m}^{2}\right)$ & $I_{0}$ & $k_{i}\left(\mathrm{KN} / \mathrm{m}^{3}\right)$ \\
\hline 1 & 300 & 12,5 & 371 \\
\hline 2 & 371 & 11,9 & 388 \\
\hline 3 & 390 & 11,7 & 392 \\
\hline
\end{tabular}

soit une valeur $\mathrm{k}$ voisine de $400 \mathrm{KN} / \mathrm{m}^{3}$ au droit des appuis profonds (majoration de $30 \%$ ).

Cette valeur s'avère notablement plus faible que celle obtenue par les règles spécifiques rapportées par Balay (1984):

cas d'un sol en place et de tirants placés sous la profondeur $1_{0}^{*} / 3$ :

$$
I_{\mathrm{o}}^{*}=\sqrt[3]{\frac{E_{b} I}{3 E_{m}}}
$$

et:

$$
k=\frac{12 E_{m}}{I_{o}^{*}}
$$

$\mathrm{E}_{\mathrm{b}} \mathrm{I}$ : produit d'inertie de l'écran pour $1 \mathrm{ml}$;

soit ici $\mathrm{k}=6000 \mathrm{kN} / \mathrm{m}^{3}$ au droit de tirants placés sous $6 \mathrm{~m}$ de profondeur;

ou $\mathrm{k}=2000 \mathrm{kN} / \mathrm{m}^{3}$ au droit de tirants proches de la surface. Une approche systématique confirme cette divergence entre les deux approches dans la zone 4. La figure 12 compare ainsi la détermination de $k$ par les relations (11) et (12) (Balay, 1984) à celle du processus itératif basé sur la relation (10) pour un module $\mathrm{E}_{\mathrm{m}}=100 \mathrm{MPa}$ et des valeurs du produit $\mathrm{E}_{\mathrm{b}}$ I couvrant largement l'éventail des valeurs applicables aux parois moulées.

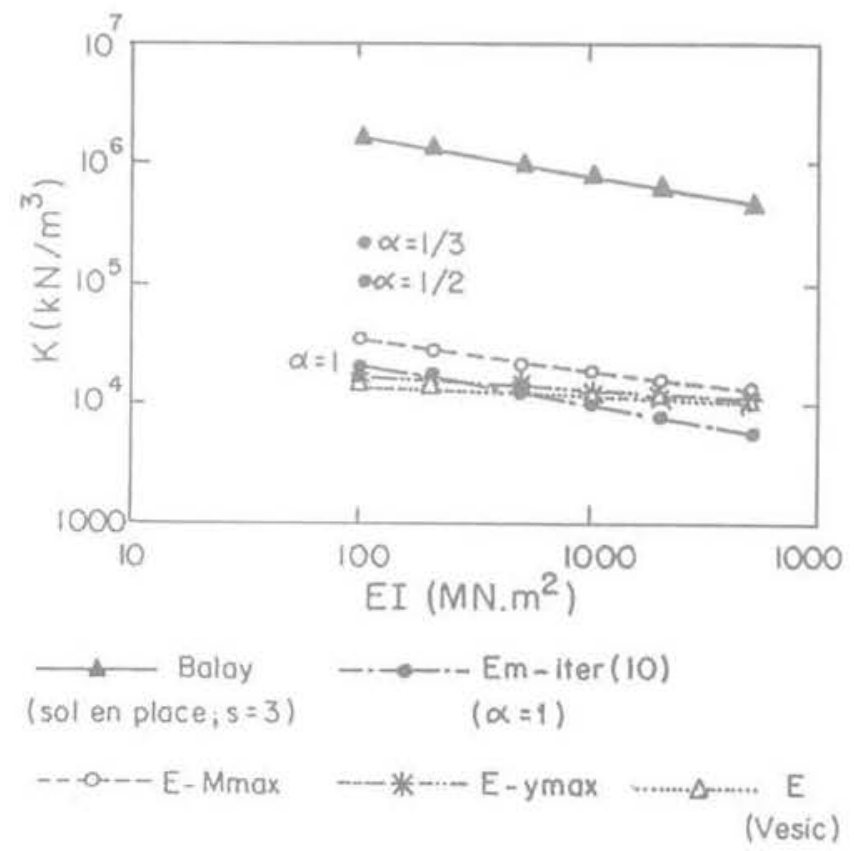

F16.12 Zone 4: Comparaison des estimations de $\mathrm{k}$ obtenues par les recommandations de Balay (1984) à celles obtenues par différents calculs fondés sur l'élasticité. Zone 4: Comparison between the $\mathrm{k}$ value estimated by the recommendations developed by Balay (1984) and those obtained by various calculations based on elasticity.

Le coefficient rhéologique $\alpha$ n'intervient que dans la relaticn (10) (et non la formulation Balay). Avec la valeur $\alpha=1$, le rapport entre k déduit des recommandations de Balay et celui obtenu de manière itérative est voisin de 100 . Il serait réduit à une valeur de l'ordre de 10 avec une valeur $\alpha=1 / 3$.

Il est intéressant de noter que trois autres calculs fondés sur l'élasticité fournissent des ordres de grandeur comparables au calcul itératif avec $\alpha=1$ (en acceptant l'équivalence $\left.\mathrm{E}_{\text {young }}=\alpha \mathrm{E}_{\mathrm{m}}\right)$ :

- a : calcul basé sur la relation (2) établie par Vesic; - b et c: calcul de l'interaction poutre-massif élastique fondé sur la solution de Boussinesq du massif isotrope et les conditions de compatibilité à l'interface poutremassif. Ce calcul établit successivement la déformée et les efforts internes $(\mathrm{M}, \mathrm{T})$ de la poutre et les réactions du massif. Les résultats peuvent ainsi être exploités en cherchant:

- soit le coefficient de proportionalité entre la réaction et le déplacement $y_{\max }$ au point de chargement $-\mathrm{k}\left(\mathrm{y}_{\max }\right)$ : calcul b;

- soit la valeur $\mathrm{k}$ du modèle à ressorts qui conduirait au même moment fléchissant maximal dans la poutre $-\mathrm{k}\left(\mathrm{M}_{\max }\right)$ : calcul c. 
Cette convergence observée sur les ordres de grandeur obtenus par des approches distinctes, nous fait considérer avec circonspection les ordres de grandeur plus élevés systématiquement obtenus par les règles LCPC applicables au droit des appuis. La confrontation rapportée par Gigan (1984) du bon accord entre cette règle et les mesures expérimentales concernait, il faut le noter, essentiellement des ouvrages en palplanches dont un placé contre un remblai où la formulation assimile $E_{m}$ et $E_{\text {young }}$ au lieu de la relation $E_{\text {young }}=3 E_{m}$ implicitement admise pour les sols en place.

En l'état actuel, une estimation de k fondée sur une approche itérative de I telle que décrite parait offrir un domaine de validité plus large que les recommandations correspondantes trouvées dans les règles LCPC.

\section{6}

\section{Conclusion}

Il importe de retenir que le coefficient de réaction n'est pas une caractéristique intrinsèque d'un sol qui ne dépendrait que de ses seules propriétés de déformation.

C'est un outil de simplification pour le projeteur dont la finalité est de rechercher et exprimer l'équivalence entre les sollicitations d'une structure considérée sur son massif de fondation lorsqu'elle est soumise à un chargement parfaitement défini (distribution et intensité) et les sollicitations de la même structure chargée lorsqu'elle est supposée placée sur une distribution continue de ressorts indépendants (modèle de Winkler).

Le coefficient de réaction n'est qu'un paramètre qui qualifie l'interaction entre une structure et sa fondation sous un chargement donné. Sa valeur dépend ainsi de la largeur de la zone sollicitée sous la structure, de la rigidité relative de celle-ci par rapport à sa fondation et des conditions aux limites du massif (caractère confiné ou non des déformations).

Une estimation raisonnée du coefficient de réaction est cependant possible si on considère effectivement l'influence de chacun cle ces facteurs.

Dans le cas des écrans de soutènement, ceci fait retenir un ensemble de règles cohérentes vis-à-vis de la formulation générale du tassement donnée par Ménard et aptes à prendre en compte la déformabilité propre de l'écran ou l'influence de la surface libre. En dehors des zones d'appuis ponctuels, ces règles reprennent pour l'essentiel les recommandations présentées par le LCPC (Balay, 1984).
Une divergence importante a été relevée dans les zones placées directement au droit d'appuis ponctuels où la formulation des règles LCPC paraît surestimer largement la raideur équivalente du massif. Une détermination itérative de la longueur de transfert $I_{\text {o }}$ nous paraît offrir un domaine de validité plus large vis-à-vis de l'éventail des rigidités couvert par les soutènements en palplanches ou paroi moulées.

On notera cependant qu'il serait illusoire de rechercher une précision excessive lors du processus itératif décrit. Il suffit de rappeler que, pour l'approche similaire que Terzaghi proposait d'appliquer aux dallages, celui-ci conseillait d'arrêter les itérations dès que l'écart entre les valeurs successives de $\mathrm{k}$ devenait inférieur à $50 \%$

L'expérience acquise tant dans le cadre de la conception des soutènements que celui d'expertises ayant à juger de certains déboires, montre que les estimations des efforts internes obtenues en incorporant les valeurs du coefficient de réaction déduites des différentes règles proposées fournissent une appréciation correcte des sollicitations réelles, au plan de la sécurité des ouvrages. Il existe plus d'incertitude quant aux estimations obtenues pour les déplacements. Une meilleure estimation passe nécessairement par une différenciation des valeurs du coefficient de réaction à utiliser aux différentes phases (notamment lors de la première phase où l'écran travaille en console ou lors de la mise en place d'appuis ponctuels éventuels, butons ou tirants). Dans tous les cas, il est recommandé de vérifier par l'examen de la déformée calculée la pertinence des choix implicites qu'expriment les paramètres (a) et (B) des relations (9) et (10). Cette vérification doit permettre de contrôler a posteriori l'influence de la rigidité de l'écran quand celle-ci r'est pas directement intégrée lors de l'estimation initiale de k (à l'inverse du calcul itératif de $\mathrm{k}$ devant les appuis).

Cette attention du projeteur à la vraisemblance des valeurs du coefficient de réaction et leur compatibilité avec la raideur de l'écran, aux conditions aux limites du massif et au type de sollicitations induites à l'interface écran-massif doit être accrue pour tous les ouvrages où des seuils de deformation sont effectivement incorporés au CCTP. Son attention devra également porter dans le cas d'ancrages actifs précontraints aux déformations éventuelles des points de scellement (calcul du soutènement composé par l'ouvrage et ses ancrages en gabion soumis à la poussée des terres) qui modifient la raideur relative des ancrages.

\section{$\overline{\text { Bibliographie }}$}

Balay-Recommandations pour le choix des paramètres de calcul des écrans de soutènement par la méthode aux modules de réaction. Note d'information technique, ministère de l'Urbanisme, du Logement et des Transports, Laboratoire Central des Ponts et Chaussées, 1984.

Biot A.M. - Bending of an infinite beam on an elastic foundation. J. Appl. Mecha. nics, 4, 1, A1-A7, 1937.

Caquot A., Kérisel J. - Traité de mécanique des sols, $4^{e}$ éd. Gauthier-Villard, 1966.

Gigan J.-P. - Expérimentation d'un rideau en palplanches ancré par tirants actifs. Bulletin de liaison des Laboratoires des
Ponts et Chaussées, 129, Janv.-Fév. 1984, pp. 5-20.

Mènard L., Bourdon G., Houy A. - Etude expérimentale de l'encastrement d'un rideau en fonction des caractéristiques pressiométriques du sol de fondation. Sols-Soils 12, 9 juin 1964.

Ménard L., Bourdon G. - Calcul des rideaux de soutènement. Méthode nouvelle prenant en compte les conditions réelles d'encadrement. Sols-Soils 12, mars 1965.

Ménard L - Règles d'utilisation des techniques pressiométriques, contraintes et déformations dans un pieu soumis à des efforts horizontaux. Notice spéciale $n^{\circ} 2$
D/62/69. Documentation $\alpha$ Technique L. Ménard x, 1973

Ménard L., Rousseau - L'évaluation des tassements, tendances nouvelles. SolsSoils, vol. I, n 1 , juin 1962, pp. 13-29.

Schmitt - Étude expérimentale de la sollicitation exercée par le sol sur les ouvrages de soutènement. Revue française de géotechnique $n^{\circ} 28,1984$, pp. $27-40$.

Terzaghi K. - Evaluation of coefficients of subgrade reaction. Geotechnique, 4, 1955, pp. 297-326.

Vesic A.B. - Bending of beams resting on isotropic elastic solid. ASCE. J. Enqineering Mechanics Division, vol.87, EM2, april 1961, pp. 35-53. 